\title{
ON THE GROTHENDIECK RING OF AN ABELIAN $p$-GOUP
}

\author{
TADAO OBAYASHI
}

\section{Introduction}

The Grothendieck ring of a finite group has been studied by Swan ([5], [6]). At the end of [6] he determined completely the structure of the Grothendieck ring $G(Z \$)$ of a cyclic $p$-group $\&$ over the ring of rational integers $Z$.

In this paper we investigate the structure of $G(Z \&)$ of an abelian $p$-group B.

In the first section we consider some properties of the integral group ring of $\mathbb{B}$. The results of this section are applied in the second section to investigate the additive structure of $G(Z \$)$. Let o be a maximal order of the group ring $Q \$ S$ over the rational number field $Q$ and let $C o(0)$ be the reduced projective class group of $\mathfrak{D}(\operatorname{Rim}[4])$. We show that $G(Z(S)$ is isomorphic to the splitting $Z$-algebra extension of $C o(0)$ by $G(Q 3)(\S 2, \S 3)$. The latter half of the third section is devoted to study the action of $G(Q \$)$ to $C o(\mathfrak{n})$. Some examples are given in the final section.

The author wishes to express his hearty thanks to Professor A. Hattori for his many helpful suggestions during the preparation of this paper.

\section{$\S 1$. The integral group ring of a finite abelian group}

Let $R$ be the ring of integers of an algebraic number field $K$. The group ring $K \mathscr{S}$ of a finite abelian group $\&$ over $K$ decomposes into a direct sum of algebraic number fields $K_{i}$ over $K$

$$
K \&=K_{1} \oplus \cdots \oplus K_{s},
$$

and $K_{1}, \ldots, K_{s}$ are a full set of non-isomorphic irreducible $K \&$-modules. This decomposition induces the decomposition of the maximal order 0 of $K \mathscr{B}$ into a direct sum of maximal orders $n_{i}$ of $K_{i}$, i.e. the ring of integers of $K_{i}$. Since

Received April 19, 1965. 
n contains $R\left(S\right.$, each projection $\pi_{i}$ of $K \&$ onto $K_{i}$ induces a ring homomorphism of $R \&$ into $D_{i}$. We will denote by $\Lambda_{i}$ the kernel of this ring homomorphism and we will set $\Gamma_{i}=\prod_{j \neq i} \Lambda_{j}$.

Proposition 1.1. Let $\$$ be a finite abelian group of order $n$ and exponent $n_{0}$ and let $K=Q\left(\zeta_{m}\right)$ be a cyclotomic field, where $\zeta_{m}$ means a primitive $m$-th root of 1 . Then

(1) in (1.1), each $K_{i}$ is also a cyclotomic field $Q\left(\zeta_{m_{i}}\right)$ for some $m_{i}$ which divides L.C.M. $\left(m, n_{0}\right)$,

(2) each projection $\pi_{i}$ induces a surjection of $R \mathbb{S}$ onto $\mathrm{D}_{i}$.

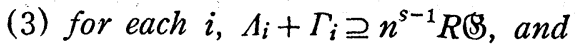

(4) there exists a positive integer $l$ such that

$$
\Gamma_{1}+\cdots+\Gamma_{s} \supseteq n^{l} R \mathbb{8} \text {. }
$$

Proof. Let $B=B_{1} \times \cdots \times B_{t}$ be the decomposition of $B$ into a direct product of cyclic subgroups $\mathbb{S}_{h}$ and let $g_{h}$ be the fixed generator of $\dot{B}_{h}$.

Then we have $K_{i}=K\left(\pi_{i}\left(g_{1}\right), \ldots, \pi_{i}\left(g_{t}\right)\right)$. But for each $h \pi_{i}\left(g_{h}\right)^{n_{0}}=1$, which implies that $K_{i}=Q\left(\zeta_{m_{i}}\right)$ for some $m_{i}$ which divides L.C.M. $\left(m, n_{0}\right)$. This shows (1). Each $\pi_{i}$ gives rise to the surjection of $R \&$ onto $R\left[\pi_{i}\left(g_{1}\right), \ldots, \pi_{i}\left(g_{t}\right)\right]$ $=Z\left[\zeta_{m_{i}}\right]$, which is the maximal order of $K_{i}=Q\left(\zeta_{m_{i}}\right)$. This proves (2). (3) and (4) is proved by an induction on $t$. First, we suppose that $\dot{\&}$ is a cyclic group generated by an element $g$. We have a ring isomorphism $K \dot{B} \cong$ $K[x] /\left(x^{n}-1\right) K[x]$, where $K[x]$ is the polynomial ring over $K$ in an indeterminate $x$. If

$$
x^{n}-1=f_{1}(x) \cdots f_{s}(x)
$$

is the factorization of $x^{n}-1$ into irreducible non-constant monic polynomials in $K[x]$, by the Chinese remainder theorem we have

$$
K[x] /\left(x^{n}-1\right) K[x] \cong K[x] / f_{1}(x) K[x] \oplus \cdots \oplus K[x] / f_{s}(x) K[x] .
$$

Obviously every root of $f_{i}(x)$ is a primitive $n_{i}$-th root of 1 for some $n_{i}$ which divides $n$. Let $\zeta_{n_{i}}$ be one of these roots and let $K_{i}=K\left(\zeta_{n_{i}}\right)$. Then the map $g \rightarrow \zeta_{n_{i}}$ gives rise to the projection $\pi_{i}$ of $K \otimes$ onto $K_{i}$. This shows that the

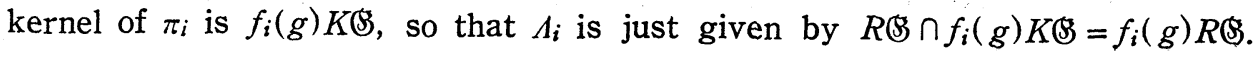
By a simple calculation, we have from (1.2) 


$$
f_{i}(x) R[x]+f_{j}(x) R[x] \supseteq n R[x] \quad(i \neq j) .
$$

Replacing $x$ by $g$, we have

$$
\Lambda_{i}+\Lambda_{j} \supseteq n R \mathbb{G} \quad(i \neq j) .
$$

This implies that $\Lambda_{i}+\prod_{j \neq i} \Lambda_{j} \supseteq n^{s-1} R \mathbb{G}$, which shows (3). (1.2) yields also that

$$
\prod_{\jmath \neq 1} f_{j}(x) R[x]+\cdots+\prod_{\jmath \neq s} f_{j}(x) R[x] \supseteq n R[x] .
$$

Since $\prod_{j \neq i} f_{j}(g) R \&=T_{i}$, this implies $(4)$.

In the general case, let $\mathbb{B}^{\prime}=\mathbb{B}_{1} \times \cdots \times \mathbb{B}_{t-1}$ and let $n^{\prime}$ and $n^{\prime \prime}$ be the order of $\mathscr{B}^{\prime}$ and $\mathbb{B}_{t}$, respectively. If $x^{n^{\prime \prime}}-1=f_{1}(x) \cdots f_{s}(x)$ is the factorization of $x^{n^{\prime \prime}}-1$ into irreducible monic polynomials in $K[x]$ and $\zeta_{n_{i}}$ is a root of $f_{i}(x)$, the map $g_{t} \rightarrow \zeta_{n_{i}}$ gives an isomorphism $K \& / f_{i}\left(g_{t}\right) K \dot{\Theta} \cong K\left(\zeta_{n_{i}}\right)\left(S^{\prime}\right.$. Denoting $K\left(\zeta_{n_{i}}\right)$ by $K_{i}$, we have $K\left(\mathbb{S} \equiv K_{1}\left(\mathbb{S}^{\prime} \oplus \cdots \oplus K_{s} \dot{\mathbb{S}}^{\prime}\right.\right.$. On the other hand, (1) implies that each $K_{i}\left(\mathbb{S}^{\prime}\right.$ is a direct sum of cyclotomic fields $K_{i, j}$ :

$$
K_{i} \mathbb{S}^{\prime}=K_{i, 1}+\cdots+K_{i, s_{i}} .
$$

Let $R_{i}$ and $\mathrm{D}_{i, j}$ be the rings of integers of $K_{i}$ and $K_{i, j}$, respectively, and let $\Lambda_{i, j}$ be the kernel of the surjection of $R \&$ onto $\mathfrak{n}_{i, j}$. This surjection is given by the combined map $R \mathbb{S} \rightarrow R_{i} \mathbb{S}^{\prime} \rightarrow 0_{i, j}$. Since $f_{i}\left(g_{t}\right) R \mathbb{S}$ is the kernel of the surjection $R \& \rightarrow R_{i} \&^{\prime}$, we see that

$$
\Lambda_{i, j} \supseteq f_{i}\left(g_{t}\right) R \otimes \quad\left(j=1, \ldots, s_{i}\right),
$$

and that the image $\bar{\Lambda}_{i, j}$ in $R_{i} \dot{\mathcal{S}}^{\prime}$ of $\Lambda_{i, j}$ is the kernel of $R_{i} \mathbb{S}^{\prime} \rightarrow \mathfrak{D}_{i, j}$. Now for any distinct $\Lambda_{i, j}$ and $\Lambda_{h, k}$, we will show that $\Lambda_{i, j}+\Lambda_{h, k} \supseteq n R \&$. When $B$ is a cyclic group, this is given in (1.5). Then for any distinct $k$ and $k^{\prime}$, the induction hypothesis shows that $\bar{\Lambda}_{i, k}+\bar{\Lambda}_{i, k^{\prime}} \supseteq n^{\prime} R_{i} \otimes^{\prime}$. Since $n^{\prime}$ divides $n$, this implies that $\Lambda_{i, k}+\Lambda_{i, k^{\prime}} \supseteq n R \&$. On the other hand, for any distinct $i$ and $i^{\prime}$, we see easily that $f_{i}\left(g_{t}\right) R \mathbb{S}+f_{i^{\prime}}\left(g_{t}\right) R \leftrightarrow\left(S \supseteq n^{\prime \prime} R\right.$ similarly as in (1.4). Since $n^{\prime \prime}$ divides $n$, (1.7) shows that $\Lambda_{i, j}+\Lambda_{i^{\prime}, j^{\prime}} \supseteq n R \Theta$. Let $\Gamma_{i, j}$ be the product of all $\Lambda_{h, k}$ but $\Lambda_{i, j}$. Then a simple calculation shows that $\Lambda_{i, j}+\Gamma_{i, j} \supseteq n^{\Sigma s_{k}-1} R \circlearrowleft$ from the above result, which proves (3). Let $\Delta_{i, j}=\prod_{k \neq j} \Lambda_{i, k}$. Then by the induction hypothesis, there exists a positive integer $l_{i}$ such that $\bar{\Delta}_{i, 1}+\cdots+\bar{\Delta}_{i, s_{i}} \supseteq n^{\prime^{\prime}} R_{i} \mathbb{S}^{\prime}$, which shows that

$$
\Delta_{i, 1}+\cdots+\Delta_{i, s_{i}} \supseteq n^{\prime l_{i}} R \mathbb{S}
$$


Since $\Lambda_{h, k}+\Lambda_{h, k} \supseteq n R \bigotimes$ for any distinct $k$ and $k^{\prime}$, it follows that $\Lambda_{h, 1} \cdots \Lambda_{h, s_{h}}$ $\supseteq n^{s_{h}\left(s_{h}-1\right) / 2}\left(\Lambda_{h, 1} \cap \cdots \cap \Lambda_{h, s_{h}}\right)$. But each $\Lambda_{h, k}$ contains $f_{h}\left(g_{t}\right) R \dot{\Theta}$ from (1.6), so that $\Lambda_{h, 1} \cdots \Lambda_{h, s_{h}} \supseteq n^{s_{h}\left(s_{h}-1\right) / 2} f_{h}\left(g_{l}\right) R \&$. Let $l^{\prime}=\operatorname{Max} .\left\{l_{1}, \ldots, l_{s}\right\}$ and $l^{\prime \prime}=$ Max. $\left\{\frac{1}{2} \sum_{h \neq 1} s_{h}\left(s_{h}-1\right), \ldots, \frac{1}{2} \sum_{h \neq s} s_{h}\left(s_{h}-1\right)\right\}$. Then we have from (1.8)

$$
\sum_{i, j} \Gamma_{i, j}=\sum_{i, j} \Delta_{i, j} \prod_{h \neq i}\left(\Lambda_{h, 1} \cdots \Lambda_{h, s_{h}}\right) \supseteq n^{l^{\prime \prime}} n^{\prime l^{\prime}} \sum_{i} \prod_{h \neq i} f_{h}\left(g_{t}\right) R \dot{8} .
$$

As in (1.5) we have $\sum_{i} \prod_{h \neq l} f_{h}\left(g_{t}\right) R \& \supseteq n^{\prime \prime} R \&$. Hence $l=l^{\prime}+l^{\prime \prime}$ satisfies (4). This completes the proof of the proposition.

\section{§2. The additive structure of $G(Z \$)$}

We are now ready to investigate the additive structure of $G(Z \mathbb{S})$ of an abelian $p$-group $\$ S$. Let $\&$ be of order $p^{e}$ and exponent $p^{e_{0}}$. We denote by $\zeta_{d}$ a primitive $p^{d}$-th root of 1 .

From Proposition 1.1, $Q \$$ is a direct sum of cyclotomic fields $K_{i}=Q\left(\zeta_{d_{i}}\right)$ for some $d_{i}$ such that $0 \leqq d_{i} \leqq e_{0}$ and the maximal order $\mathfrak{0}$ of $Q \mathbb{S}$ is also a direct sum of the maximal orders $\mathfrak{o}_{i}=Z\left[\zeta_{d_{i}}\right]$ of $K_{i}$. Furthermore, the surjection of $Z \&$ onto $\mathfrak{D}_{i}$ induced by $\pi_{i}$ gives a ring isomorphism

$$
Z \$ / \Lambda_{i} \cong 0_{i} \text {. }
$$

Let $M$ be any regular (i.e. finitely generated and $Z$-torsion free) $Z \$$-module and let

$$
M_{i}=\left\{m \in M: \lambda_{i} m=0 \text { for any } \lambda_{i} \in \Lambda_{i}\right\} .
$$

Then $M_{i}$ is a $Z$-pure submodule of $M$. Since $\Lambda_{i}$ annihilates $M_{i}$, we may turn $M_{i}$ into an $\mathfrak{D}_{i}$-module from (2.1). Clearly $M_{i}$ is finitely generated and torsion free as an $\mathfrak{D}_{i}$-module, so that $M_{i}$ is projective since $o_{i}$ is a Dedekind ring. Thus $M_{i}$ is isomorphic to the direct sum of $l_{i}-1$ copies of $D_{i}$ and an ideal a of $\mathrm{D}_{i}$

$$
M_{i} \cong \mathfrak{o}_{i} \oplus \cdots \oplus \mathfrak{o}_{i} \oplus \mathfrak{a}
$$

where the $o_{i}$-rank $l_{i}$ of $M_{i}$ and the ideal class $C_{i}(a)$ of $a$ are complete invariants of $M_{i}$ (Curtis and Reiner [3]). By Proposition 1.1, (3), we have $M_{i} \cap\left(M_{1}+\right.$ $\left.\cdots+M_{i-1}+M_{i+1}+\cdots+M_{s}\right)=0$. This shows that the sum of $M_{i}$ is a direct sum. Now we denote by $\bar{M}$ the quotient $M / \sum \oplus M_{i}$. Since $\Lambda_{i} \Gamma_{i}=0, \bar{M}$ is annihilated by $I_{i}+\cdots+\Gamma_{s}$. Then Proposition 1.1, (4) implies that $\bar{M}$ may be regarded as a module over $Z /\left(p^{e l}\right) \&$ for some positive integer $l$. But the 
only irreducible $Z /\left(p^{e l}\right) \&$-module is $Z /(p)$ on which $\$$ acts trivially. Hence $\bar{M}$ has a composition series with factors $Z /(p)$. The sequence

$$
0 \rightarrow Z \stackrel{p}{\rightarrow} Z \longrightarrow Z /(p) \rightarrow 0
$$

shows that $[Z /(p)]=0$ in $G(Z \&)$, where $[Z /(p)]$ means the element of $G(Z \&)$ associated with $Z /(p)$, so that $[\bar{V}]=0$ in $G(Z \mathbb{B})$. This implies that $[M]=\sum\left[M_{i}\right]$. For any ideal $a$ of $n_{i}$ we denote by $a_{i}^{*}$ the element $[\mathfrak{a}]-\left[0_{i}\right]$ of $G(Z \mathbb{S})$. The map $a \rightarrow a_{i}^{*}$ defines a homomorphism of the ideal class group of $\mathfrak{n}_{i}$ to $G(Z \mathbb{S})$, and from (2.2), any element $x$ of $G(Z \&)$ may be written in the form

$$
x=\sum_{i}\left(l_{i}\left[0_{i}\right]+\mathfrak{a}_{i}^{*}\right) \quad\left(l_{i} \in Z\right) .
$$

The uniqueness of this expression follows immediately from the following proposition.

Proposition 2.1. For any exact sequence of regular Z\$S-modules

$$
0 \longrightarrow M^{\prime} \longrightarrow M \stackrel{\psi}{\rightarrow} M^{\prime \prime} \longrightarrow 0
$$

we have $C_{i}(\mathfrak{a})=C_{i}\left(\mathfrak{a}^{\prime}\right) \cdot C_{i}\left(\mathfrak{a}^{\prime \prime}\right)$, where $C_{i}(\mathfrak{a}), C_{i}\left(\mathfrak{a}^{\prime}\right)$ and $C_{i}\left(\mathfrak{a}^{\prime \prime}\right)$ are ideal class invariants of $M_{i}, M_{i}^{\prime}$ and $M_{i}^{\prime \prime}$, respectively.

Proof. The sequence (2.3) induces an exact sequence

$$
0 \rightarrow \operatorname{Hom}_{z \mathscr{S}}\left(\mathrm{o}_{i}, M^{\prime}\right) \rightarrow \operatorname{Hom}_{z \mathfrak{S}}\left(\mathrm{o}_{i}, M\right) \rightarrow \operatorname{Hom}_{z \mathfrak{G}}\left(\mathrm{o}_{i}, M^{\prime \prime}\right) \rightarrow \operatorname{Ext}_{z \mathscr{S}}^{1}\left(\mathrm{o}_{i}, M^{\prime}\right) .
$$

But $\operatorname{Hom}_{z \mathscr{S}}\left(\mathfrak{o}_{i}, M\right)$ is isomorphic to $M_{i}$ by the map $f \rightarrow f(1)$. Hence we have an exact sequence

$$
0 \rightarrow M_{i}^{\prime} \rightarrow M_{i} \rightarrow M_{i}^{\prime \prime} \rightarrow \operatorname{Ext}_{z \mathbb{S}}^{1}\left(\mathfrak{o}_{i}, M^{\prime}\right) .
$$

Since the order $p^{e}$ of $\left(\mathbb{S}\right.$ annihilates $\operatorname{Ext}_{Z \mathbb{S}}^{1}\left(0_{i}, M^{\prime}\right)$ (Cartan and Eilenberg [2]), we see that

$$
p^{e} M_{i}^{\prime \prime} \subseteq \psi\left(M_{i}\right) \subseteq M_{i}^{\prime \prime}
$$

where $\phi\left(M_{i}\right)$ is also a projective $\mathfrak{n}_{i}$-module whose $\mathfrak{n}_{i}$-rank is equal to that $M_{i}^{\prime \prime}$. Thus by Invariant factor theorem ([3]), there exist elements $u_{1}, \ldots, u_{l_{i}}$ of $M_{i}^{\prime \prime}$ and ideals $\mathfrak{b}_{1}, \ldots, \mathfrak{b}_{l_{i}}$ of $\mathfrak{b}_{i}$ such that

$$
\begin{aligned}
& M_{i}^{\prime \prime}=\mathfrak{o}_{i} u_{1} \oplus \cdots \oplus \mathfrak{b}_{i} u_{l_{i}-1} \oplus \mathfrak{a}^{\prime \prime} u_{l_{i}} \\
& \psi\left(M_{i}\right)=\mathfrak{b}_{1} u_{1} \oplus \cdots \oplus \mathfrak{b}_{l_{i}-1} u_{l_{i}-1} \oplus \mathfrak{b}_{l_{i}} \mathfrak{a}^{\prime \prime} u_{l_{i}} .
\end{aligned}
$$


Then the inclusion (2.4) shows that each $\mathfrak{b}_{k}$ divides $\left(p^{e}\right)$. But $p$ is a power of the principal prime ideal $\left(1-\zeta_{d_{i}}\right)$ of $\mathfrak{b}_{i}$, which implies that $b_{k}$ is also a principal ideal. Then $C_{i}\left(\mathfrak{b}_{1} \cdots \mathfrak{b}_{l_{i}} \mathfrak{a}^{\prime \prime}\right)=C_{i}\left(\mathfrak{a}^{\prime \prime}\right)$. Furthermore, $M_{i}$ is isomorphic to the direct sum of $M_{i}^{\prime}$ and $\psi\left(M_{i}\right)$ since $\phi\left(M_{i}\right)$ is projective. Therefore $C_{i}(a)=$ $C_{i}\left(\mathfrak{a}^{\prime}\right) \cdot C_{i}\left(\mathfrak{b}_{1} \cdots \mathfrak{b}_{l_{i}} \mathfrak{a}^{\prime \prime}\right)$, which coincides with $C_{i}\left(\mathfrak{a}^{\prime}\right) \cdot C_{i}\left(\mathfrak{a}^{\prime \prime}\right)$. This completes the proof.

THEOREM 2.1. If $\&$ is an abelian p-group, $G(Z \$)$ is isomorphic to the direct sum of $C_{0}(0)$ and $G(Q \$)$ as an additive group

$$
G(Z \mathbb{B}) \cong C_{0}(\mathrm{0}) \oplus G(Q(\mathbb{S}) .
$$

Proof. Since $\mathrm{D}$ is the direct sum of the $\mathrm{o}_{i}, C_{0}(\mathrm{o})=\sum \oplus C_{0}\left(\mathrm{o}_{i}\right)$ and each $C_{0}\left(\mathrm{o}_{i}\right)$ is isomorphic to the ideal class group of $\mathrm{o}_{i}(\operatorname{Rim}[4])$. Then the map $C_{i}(\mathfrak{a}) \rightarrow a_{i}^{*}$ defines a homomorphism $\phi: C_{0}(\mathfrak{b}) \rightarrow G(Z \dot{B})$, where the action of $\mathbb{B}$ on $a$ is given by setting $g \cdot \alpha=\pi_{i}(g) \alpha, g \in \mathbb{B}, \alpha \in \mathfrak{a}$. On the other hand, $\left[K_{1}\right], \ldots$, $\left[K_{s}\right]$ make a base for $G(Q \mathbb{S})$. We define a linear map $\varphi: G(Q \&) \rightarrow G(Z \mathbb{S})$ by $\varphi\left(\left[K_{i}\right]\right)=\left[0_{i}\right]$. Then we have an additive isomorphism $C_{0}(0) \oplus G(Q \mathbb{B}) \rightarrow G(Z \mathbb{B})$ by $(x, y) \rightarrow \phi(x)+\varphi(y)$ because the image $\phi(x)+\varphi(y)$ in $G(Z \&)$ is uniquely determined by Proposition 2.1. This proves Theorem 2.1.

\section{§ 3. Ring structure}

We will now study the multiplicative structure of $G(Z \$)$. In (2.5), Swan [6] showed that $\phi\left(C_{0}(0)\right)^{2}=0$. Hence $G(Z \dot{S})$ is a $Z$-algebra extension over an abelian kernel, and is determined by the action of $G\left(Q(B)\right.$ to $C_{0}(0)$ and the associated 2-cohomology class of $H^{2}\left(G(Q \&), C_{0}(0)\right)$.

In this section we denote by $p^{e_{h}}$ the order of a cyclic factor $\mathbb{B}_{h}$ of $\mathbb{B}$. As in $\S 2$, each $\pi_{i}\left(g_{h}\right)$ is of the form $\zeta_{d_{i}}^{i_{h}}$ for some integer $i_{h}$ such that $0 \leqq i_{h} \leqq e_{0}$, which satisfies $i_{h} p^{e_{h}} \equiv 0\left(\bmod p^{d_{i}}\right)$. In general, given a $t$-tuple $\left(\xi_{1}, \ldots, \xi_{t}\right)$ of integers which satisfy that $\xi_{h} p^{e_{h}} \equiv 0\left(\bmod p^{\lambda_{i}}\right)$ for each $h$, we may construct a regular $Z \dot{S}$-module as follows. Let $a$ be an ideal of $Z\left[\zeta_{d_{i}}\right]$. We turn $a$ into a regular $Z \mathbb{B}$-module by defining

$$
g_{h} \cdot \alpha=\zeta_{a_{2}}^{\xi_{h}} \alpha, \alpha \in \mathfrak{a} .
$$

We denote this module by $\left(a ; \xi_{1}, \ldots, \xi_{t}\right)$. In particular, for the $t$-tuple $\left(i_{1}, \ldots, i_{t}\right), i_{h}$ being as above, we denote $\left(a ; i_{1}, \ldots, i_{t}\right)$ by $a_{i}$. Then the element $a_{i}^{*}$ of $G(Z \&)$ can be written in the form $\left[a_{i}\right]-\left[0_{i}\right]$. 
Proposition 3.1. For any ideal a of $Z\left[\zeta_{d_{i}}\right],\left(\mathfrak{a} ; \xi_{1}, \ldots, \xi_{t}\right)$ is reducible if and only if every $\xi_{h}$ is divisible by $p$.

Proof. $\left(a ; \xi_{1}, \ldots, \xi_{t}\right)$ is reducible if and only if $Q \otimes_{z}\left(a ; \xi_{1}, \ldots, \xi_{t}\right)$ is reducible. Let $Q \otimes_{z}\left(a, \xi_{1}, \ldots, \xi_{t}\right)$ be reducible. Then this contains, as a direct summand, $K_{j}$ for some $j$ such that $d_{j}<d_{i}$ and each $g_{h}$ acts on $K_{j}$ as the multiplication of $\zeta_{d_{i}}^{\xi_{h}}$. This shows that every $\xi_{h}$ is divisible by $p$. Conversely let every $\xi_{h}$ be divisible by $p$ and let $p^{d_{i}-d_{j}}$ be the highest power of $p$ which divides every $\xi_{h}$. Set $\xi_{h}=\xi_{h}^{\prime} \cdot p^{d_{i} \sim d_{j}}$. Then $Q \otimes_{2}\left(Z\left[\zeta_{d_{j}}\right]: \xi_{1}^{\prime}, \ldots, \xi_{t}^{\prime}\right)$ is obviously a direct summand of $Q \otimes_{z}\left(a ; \xi_{1}, \ldots, \xi_{t}\right)$. This proves the proposition.

PROPOsition 3.2. Let a be any ideal of $Z\left[\zeta_{d_{i}}\right]$. If $\left(a ; \xi_{1}, \ldots, \xi_{t}\right)$ is irreducible, there exist some $j$ and an ideal $\mathfrak{b}$ of $Z\left[\zeta_{d_{j}}\right]$ such that $d_{j}=d_{i}$ and $\left(a ; \xi_{1}, \ldots, \xi_{t}\right)$ $\cong \mathfrak{b}_{j}$ as $Z \dot{S}$-modules. Otherwise, there exist some $j$ and an ideal $\mathfrak{b}$ of $Z\left[\zeta_{d}\right]$ such that $d_{j}<d_{i}$ and $\left(a ; \zeta_{1}, \ldots, \xi_{t}\right) \cong \mathfrak{p}_{j} \oplus \cdots \oplus \mathfrak{o}_{j} \oplus \mathfrak{b}_{j}\left(p^{d_{i}-d_{j}}\right.$ summands $)$ as $Z \mathbb{S}$. modules.

Proof. Let $\left(a ; \xi_{1}, \ldots, \xi_{t}\right)$ be irreducible. Then this is annihilated by only one $\Lambda_{j}$, so that this can be regarded as an $\mathfrak{o}_{j}$-module as in $\S 2$. By the irreducibility, $\left(\mathfrak{a} ; \xi_{1}, \ldots, \xi_{t}\right)$ is, then, isomorphic to some $\mathfrak{b}_{j}$. Hence the $Z$ rank of $\mathrm{o}_{j}$ is equal to that of $\mathfrak{o}_{i}$, and we have $d_{j}=d_{i}$. This proves the first assertion.

Let $\left(a ; \xi_{1}, \ldots, \xi_{t}\right)$ be reducible. Then each $\xi_{h}$ is divisible by $p$ (Proposition 3.1.). Let $p^{d_{i}-d_{j^{\prime}}}$ be the highest power of $p$ which divides every $\xi_{h}$ and let $\xi_{h}=\xi_{h}^{\prime} \cdot p^{d_{i}-d_{j^{\prime}}}$. Then each $g_{h}$ acts on $\left(a ; \xi_{1}, \ldots, \xi_{t}\right)$ as the multiplication of $\xi_{d_{i}}^{\xi}=\zeta_{j_{j^{\prime}}}^{\hbar h^{\prime}}$. Since $\mathfrak{a}$ is, as a $Z\left[\zeta_{d_{j^{\prime}}}\right]$-module, finitely generated and projective, $a$ is isomorphic to the direct sum of $p^{d_{i}-d_{j^{\prime}}}-1$ copies of $Z\left[\zeta_{d_{j^{\prime}}}\right]$ and an ideal $\mathfrak{b}^{\prime}$ of $Z\left[\zeta_{d_{j}}\right]$. Then we have a $Z \$$ S-isomorphism

$$
\begin{gathered}
\left(a ; \xi_{1}, \ldots, \xi_{t}\right) \cong\left(Z\left[\zeta_{d_{g^{\prime}}}\right] ; \xi_{1}^{\prime}, \ldots, \xi_{t}^{\prime}\right) \oplus \cdots \oplus\left(Z\left[\zeta_{d^{\prime}}\right] ; \xi_{1}^{\prime}, \ldots, \xi_{t}^{\prime}\right) \\
\oplus\left(\mathfrak{b}^{\prime} ; \xi_{1}^{\prime}, \ldots, \xi_{t}^{\prime}\right),
\end{gathered}
$$

where each summand is irreducible. Hence, there exist some $j$ and ideals $c$ and $\delta$ such that $d_{j}=d_{j^{\prime}},\left(Z\left[\zeta_{d_{j^{\prime}}}\right]: \xi_{1}^{\prime}, \ldots, \xi_{t}^{\prime}\right) \cong c_{j}$ and $\left(\mathfrak{b}^{\prime} ; \xi_{1}^{\prime}, \ldots, \xi_{t}^{\prime}\right) \cong \delta_{j}$

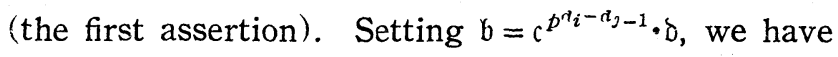

$$
\left(a ; \xi_{1}, \ldots, \xi_{t}\right) \cong \mathfrak{o}_{j} \oplus \cdots \oplus \mathfrak{o}_{j} \oplus \mathfrak{b}_{j} .
$$


This proves the second assertion and completes the proof of the proposition.

Corollary 3.1. If $\left(Z\left[\xi_{d_{i}}\right] ; \xi_{1}, \ldots, \xi_{t}\right)$ is irreducible, there exists some $j$ such that $d_{j}=d_{i}$ and $\left(Z\left[\zeta_{d_{i}}\right] ; \xi_{1}, \ldots, \xi_{l}\right) \cong 0_{j}$. Otherwise, there exists some $j$ such that $d_{j}<d_{i}$ and $\left(Z\left[\zeta_{d_{j}}\right] ; \xi_{1}, \ldots, \xi_{t}\right) \cong \mathfrak{n}_{j} \oplus \cdots \oplus \mathfrak{p}_{j}\left(p^{d_{i}-d_{j}}\right.$ summands $)$.

Proof. According to Artin [1] $(D / \Delta)^{1 / 2}$ is the ideal class invariant of $Z\left[\zeta_{d_{i}}\right]$ as a $Z\left[\zeta_{d_{j}}\right]$-module, where $D$ is the discriminant of $Z\left[\zeta_{d_{i}}\right]$ over $Z\left[\zeta_{d_{j},}\right]$ and $\Delta$ is the discriminant of any equation defining the extension of $Q\left(\zeta_{d_{i}}\right)$ over $Q\left(\zeta_{d_{j^{\prime}}}\right)$. But it is easily checked that $(D / \Delta)^{1 / 2}$ divides some power of $p$. Then $(D / \Delta)^{1 / 2}$ is a principal ideal. Hence, by Proposition 3.2, it is sufficient to prove that $b$ is a principal ideal. Let $\tau$ be the isomorphism $\left(Z\left[\zeta_{d_{i}}\right] ; \xi_{1}, \ldots, \xi_{t}\right) \cong \mathfrak{b}_{j}$. Since $Z\left[\zeta_{d_{i}}\right]$ is generated by $1, \mathfrak{b}$ is generated by $\tau(1)$. This shows that $\mathfrak{b}$ is a principal ideal, which completes the proof.

Proposition 3.3. Let a be any ideal of $Z\left[\zeta_{d_{i}}\right]$ and let $\sigma$ be a Galois automorphism of $Q\left(\zeta_{d_{i}}\right)$. If $\zeta_{d_{i}}^{\sigma}=\zeta_{d_{i}}^{\nu}$, then

$$
\left(a ; \xi_{1}, \ldots, \xi_{t}\right) \cong\left(a^{a} ; \xi_{1} \nu, \ldots, \xi_{t} \nu\right) .
$$

Proof. This follows immediately from the comparison of actions of $\&$ to the both sides.

LEMMA 3.1. If $d_{i} \geqq d_{j}$, then for any ideal a of $Z\left[\zeta_{d_{i}}\right]$ we have

$$
\left[\mathfrak{o}_{j}\right]\left[\mathfrak{a}_{i}\right]=\sum_{\sigma \mathcal{E} \in \dot{\theta}_{t} j_{j}}\left[\left(\mathfrak{a}: i_{1}+j_{1} \nu p^{d_{i}-d_{j}}, \ldots, i_{t}+j_{t} \nu p^{d_{i}-d_{j}}\right)\right],
$$

where $G_{d_{j}}$ denotes the Galois group of $Q\left(\zeta_{d_{j}}\right)$ and $\sigma_{\downarrow}$ denotes an element of $G_{d_{j}}$ such that $\zeta_{d_{j}}^{x_{j}}=\zeta_{d_{j}}^{\nu}$.

Proof. Let $\Phi_{d,}(x)$ be the cyclotomic polynomial of index $p^{d_{j}}$. Then we have $\mathrm{o}_{j} \cong Z[x] / \Phi_{d_{j}}(x) Z[x]$. This implies the isomorphism

$$
\mathrm{D}_{j} \otimes{ }_{z} \mathfrak{a}_{i} \cong \mathrm{a}[x] / \Phi_{d_{j}}(x) \mathrm{a}[x] .
$$

Let $M=a_{i}[x] / \Phi_{d}(x) \mathfrak{a}_{i}[x]$. $\quad \dot{S}$ operates on $M$ by $g_{h} m=\zeta_{d_{i}}^{i_{h}} x^{j_{h}} \cdot m, m \in M$. The assumption $d_{i} \geqq d_{j}$ implies that $\emptyset_{d_{j}}(x)$ factorizes into $\prod_{v \sim \in \sigma_{d_{j}}}\left(x-\zeta_{d}^{\nu}\right)$ in $\mathfrak{0}_{i}[x]$. Let $\sigma_{\nu_{1}}, \ldots, \sigma_{\nu_{l}}$ be the elements of $G_{d_{j}}$ and let $M_{k}=\left(x-\zeta_{d_{j}}^{\nu_{2}}\right) \cdots\left(x-\zeta_{d_{j}}^{\nu_{k}}\right) M$. Then we have a series of submodules of $M$

$$
M \supseteq M_{1} \supseteq \cdots \supseteq M_{l}=0 .
$$


Each quotient $M_{k-1} / M_{k}$ is $a_{i}[x] /\left(x-\zeta_{d_{j}}^{\nu_{k}}\right) \mathfrak{a}_{i}[x]$, which is isomorphic to a by the map $x \rightarrow \zeta_{d j}^{\nu_{c}}$. But this map carries $\zeta_{d i}^{i_{h}} x^{j_{h}}$ into $\zeta_{d i}^{i_{h}} \zeta_{d j}^{j_{h \nu_{k}}}=\zeta_{d_{i}}^{i_{h}+j_{h_{k} \nu_{k}} p r_{i}-d_{j}}$. Then each $M_{k-1} / M_{k}$ is, as a $Z\left(S\right.$-module, isomorphic to $\left(\mathfrak{a}: i_{1}+j_{1} \nu_{k} p^{d_{i}-d_{j}}, \ldots, i_{t}+j_{t \nu k} p^{d_{i}-d_{j}}\right)$. Since $M$ is composed from these modules by forming extensions, we conclude that

$$
[M]=\sum_{\sigma \in G_{d^{\prime} j}}\left[\left(a: i_{1}+j_{1} \nu p^{d_{t}-d_{j}}, \ldots, i_{t}+j_{t} \nu p^{d_{i}-d_{j}}\right)\right]
$$

This proves the lemma.

Now we will prove that $Z$-algebra extension (2.5) splits.

Theorem 3.1. The linear map $\varphi$ defined in the proof of Theorem 2.1 is a ring homomorphism. Hence the Z-algebra extension (2.5) splits.

Proof. Take any two generators $\left[K_{i}\right]$ and $\left[K_{j}\right]$ of $G(Q \$)$. We may assume that $d_{i} \geqq d_{j}$. From Lemma 3.1, we have

$$
\left[0_{j}\right]\left[n_{i}\right]=\sum_{\sigma \nu \in G_{i d_{j}}}\left[\left(Z\left[\zeta_{d_{i}}\right]: i_{1}+j_{1} \nu p^{d_{i}-d_{j}}, \ldots, i_{t}+j_{t} \nu p^{d_{i}-d_{j}}\right)\right] .
$$

But each term of the right hand is equal to either $\left[0_{k}\right]$ for some $k$ such that $d_{k}=d_{i}$ or a direct sum of $p^{d_{i}-d_{k^{\prime}}}$ copies of $\left[\mathrm{o}_{k^{\prime}}\right]$ for some $k^{\prime}$ such that $d_{k^{\prime}}<d_{i}$ (Corollary 3.1). Then we have

$$
\left[\mathrm{o}_{j}\right]\left[\mathrm{o}_{i}\right]=\sum_{\substack{k \\ d_{k}=d_{i}}}\left[\mathrm{o}_{k}\right]+\sum_{\substack{k^{\prime} \\ d k^{\prime}<a_{i}}} p^{d_{i}-d_{h^{\prime}}}\left[\mathrm{o}_{k^{\prime}}\right] .
$$

This shows that $\varphi$ is a ring homomorphism, and this completes the proof of Theorem 3.1.

LeмmA 3.2. If $d_{i} \leqq d_{j}$, then for any ideal a of $Z\left[\zeta_{d_{i}}\right]$

$$
\left[\mathrm{o}_{j}\right]\left[\mathfrak{a}_{i}\right]=\sum_{\Omega_{\nu} \in \dot{G}_{d_{i}}}\left[\left(\ddot{a}: i_{1} p^{d_{j}-d_{i}}+j_{1} \nu, \ldots, i_{t} p^{d_{j}-d_{i}}+j_{t \nu}\right)\right],
$$

where ã denotes $a Z\left[\zeta_{d_{j}}\right]$.

Proof. Notice that if $d_{i} \leqq d_{j}$, the cyclotomic polynomial $\Phi_{d_{j}}(x)$ factorizes into $\prod_{\nu, G_{a_{i}}}\left(x^{p^{d_{j}-a_{i}}}-\zeta_{d_{i}}^{\prime}\right)$ in $0_{i}[x]$ and $\zeta_{d_{j} j}^{\nu}$ is a root of $x^{p^{d_{j}-a_{i}}}-\zeta_{d i}^{\nu}$. Then the lemma is proved by the same method as the proof of Lemma 3.1.

Let $a$ be any ideal of $Z\left[\zeta_{d_{i}}\right]$ and $\left(\xi_{1}, \ldots, \xi_{t}\right)$ be any $t$-tuple of integers such that $\xi_{h} p^{e_{h}} \equiv 0\left(\bmod p^{d_{i}}\right)$. We denote the element $\left[\left(a ; \xi_{1}, \ldots, \xi_{t}\right)\right]-$ $\left[\left(Z\left[\zeta_{d_{l}}\right] ; \xi_{1}, \ldots, \xi_{t}\right)\right]$ by $\left(\mathfrak{a} ; \xi_{1}, \ldots, \xi_{t}\right)^{*}$. Then $\left(\mathfrak{a} ; \xi_{1}, \ldots, \xi_{t}\right)^{*}$ is obviously 
contained in $\phi\left(C_{0}(\mathrm{o})\right)$.

THEOREM 3.2. For any $\mathfrak{a}_{i}^{*}$ of $\phi\left(C_{0}\left(\mathfrak{D}_{i}\right)\right)$, each generator $\left[K_{j}\right]$ of $G(Q \$ S)$ acts on $\mathrm{a}_{i}^{*}$ as follows.

$$
\left[K_{j}\right] \mathrm{a}_{i}^{*}=\left\{\begin{array}{l}
\sum_{\sigma \nu \in G_{d_{j}}}\left(a: i_{1}+j_{1} \nu p^{d_{i}-d_{j}}, \ldots, i_{t}+j_{t} \nu p^{d_{i}-d_{j}}\right)^{*} \text { if } d_{i} \geqq d_{j} . \\
\sum_{\sigma \nu \in \sigma_{\sigma_{i}}}\left(\widetilde{a}: i_{1} p^{d_{j}-d_{i}}+j_{1} \nu, \ldots, i_{t} p^{d_{j}-d_{i}}+j_{t}\right)^{*} \text { if } d_{i} \leqq d_{j} .
\end{array}\right.
$$

Proof. The action of $\left[K_{i}\right]$ on $\phi\left(C_{0}(0)\right)$ is given by the multiplication of $\varphi\left(\left[K_{j}\right]\right)=\left[0_{j}\right]$. Then this theorem follows immediately from preceding two lemmas.

\section{§ 4. Example}

Let $\left(\mathcal{S}\right.$ be an abelian group of type $\left(p, p^{e}\right)$, that is, $\mathbb{S}$ be a direct product of cyclic groups $\mathbb{B}_{1}=\left(g_{1}\right)$ and $\mathbb{B}_{2}=\left(g_{2}\right)$ of order $p$ and $p^{e}$, respectively. In this case we can describe more explicitly the action of $G(Q \otimes)$ to $\left(C_{0}(0)\right)$. In this section we denote by $\zeta_{i}$ a primitive $p^{i}$-th root of 1 for any integer $i$ such that $1 \leqq i \leqq e$.

Let $a$ be any ideal of $Z\left[\zeta_{i}\right]$ and let $\nu$ be any integer such that $0 \leqq \nu \leqq p-1$. We denote $\left(a: p^{i-1} \nu, 1\right)$ by $a_{i, \nu}$ Put $o_{i, \nu}=\left(Z\left[\zeta_{i}\right]\right)_{i, \nu}$ and $K_{i, \nu}=Q \otimes_{z} \mathrm{D}_{i, \nu}$ Furthermore; for any ideal $a$ of $Z\left[\zeta_{1}\right]$ we denote $(a: 1,0)$ by $a_{0}$. Put $n_{0}=$ $\left(Z\left[\zeta_{1}\right]\right)_{0}$ and $K_{0}=Q \otimes_{2} D_{0}$. Then we see that

$$
Q \mathbb{Q B}=Q \oplus K_{0} \oplus \sum_{i=1}^{e} \sum_{\nu=0}^{p-1} K_{i, \nu}
$$

and that

$$
C_{0}(\mathfrak{D})=C_{0}\left(\mathrm{o}_{0}\right) \oplus \sum_{i=1}^{e} \sum_{\nu=0}^{p-1} C_{0}\left(\mathrm{o}_{i, \nu}\right) .
$$

1. $[Q]$ acts on $\phi\left(C_{0}(\mathfrak{D})\right)$ trivially.

2. The action of $\left[K_{0}\right]$ on $\phi\left(C_{0}\left(D_{0}\right)\right)$.

For any element $a_{0}^{*}$ of $\phi\left(C_{0}\left(D_{0}\right)\right)$ it follows immediately from Theorem 3.2 and Proposition 3.3 that

$$
\begin{aligned}
{\left[K_{0}\right] a_{0}^{*} } & =\sum_{\alpha \in G_{1}}(a ; 1+\mu, 0)^{*} \\
& =\sum_{\substack{\sigma \mu \in G_{1} \\
\mu \neq-1(\bmod p)}}\left(\mathfrak{a}^{\sigma_{1+\mu}^{-1}} ; 1,0\right)^{*}+(\mathfrak{a} ; 0,0)^{*}=\sum_{\substack{\sigma \in \in\left(\xi_{1} \\
\mu \neq-1(\bmod p)\right.}}\left(\mathfrak{a}^{\left.\sigma_{1+\mu}^{-1}\right)_{0}^{*}}\right.
\end{aligned}
$$

since $(a: 0,0)^{*}=(Z: \dot{0}, 0)^{*}=0$ by Proposition 3.2. On the other hand, $\sigma_{1+\mu}^{-1}$ such that $\mu \neq-1(\bmod p)$ ranges over all elements of $G_{1}$ but $\sigma_{1}$. Then 
$\prod_{\substack{\alpha \mu \in G_{1} \\ \mu \neq-1(\bmod p)}} \mathfrak{a}^{\sigma_{1+\mu}^{-1}}=N_{1 / 0}(\mathfrak{a}) \mathfrak{a}^{-1}$, where $N_{i / 0}$ means the norm of $Z\left[\zeta_{i}\right]$ over $Z$. Since $N_{i / 0}(a)$ is a principal ideal, $\left(N_{i / 0}(\mathfrak{a})\right)_{0}^{*}=0$. Hence we conclude that

$$
\left[K_{0}\right] a_{0}^{*}=-a_{0}^{*} .
$$

3. The action of $\left[K_{0}\right]$ on $\phi\left(C_{0}\left(o_{i}\right)\right)$.

It follows immediately from Theorem 3.2 that

$$
\left[K_{0}\right] a_{i, \nu}^{*}=\sum_{\sigma \mu \in G_{1}}\left(a: p^{i-1}(\nu+\mu), 1\right)^{*},
$$

where $\nu+\mu$ ranges over $0,1, \ldots, \nu-1, \nu+1, \ldots, p-1 \bmod p$. Hence,

$$
\left[K_{0}\right] \mathrm{a}_{i, \nu}^{*}=\sum_{\mu=0, \mu \neq \nu}^{p-1} a_{i, \mu}^{*} .
$$

4. The action of $\left[K_{i, \nu}\right]$ on $\phi\left(C_{0}\left(D_{0}\right)\right)$.

Let $x_{\mu}$ be an integer such that $\mu x_{\mu} \equiv 1\left(\bmod p^{i}\right)$. Then Theorem 3.2 and Proposition 3.3 imply that

$$
\left[K_{i, \nu}\right] a_{0}^{*}=\sum_{\sigma \mu \in G_{1}}\left(\tilde{a} ; p^{i-1}(1+\nu \mu), \mu\right)^{*}=\sum_{\sigma \mu \in G_{1}}\left(\tilde{a}^{-1} ; p^{i-1}\left(x_{\mu}+\nu\right), 1\right)^{*} .
$$

But we can easily check that $x_{\mu}+\nu$ ranges over $0,1, \ldots, p-1 \bmod p$. Hence we have

$$
\left[K_{i, \nu}\right] a_{0}^{*}=\sum_{\sigma_{\mu} \in G_{1}}\left(\widetilde{a}^{\sigma_{\mu}}\right)_{i, \nu+\mu}^{*} .
$$

5. The action of $\left[K_{j, v}\right]$ on $\phi\left(C_{0}\left(0_{i, v}\right)\right)$.

The case $i>j$. Let $y_{\mu}$ be an integer such that $\left(1+p^{i-j} \mu\right) y_{\mu} \equiv 1\left(\bmod p^{i}\right)$. Then Theorem 3.2 and Proposition 3.3 imply that

$$
\begin{aligned}
{\left[K_{j, \nu}\right] a_{i, \nu^{\prime}}^{*} } & =\sum_{\sigma \mu \in G_{j}}\left(a ; p^{i-1}\left(\nu^{\prime}+\nu \mu\right), 1+p^{i-j} \mu\right)^{*} \\
& =\sum_{\sigma \mu \in G_{j}}\left(a^{\sigma_{\mu}} ; p^{i-1}\left(\nu^{\prime}+\nu \mu\right), 1\right)^{*}
\end{aligned}
$$

because $y_{\mu} \equiv 1(\bmod p)$. In general we denote by $G_{i / j}$ the Galois group of $Q\left(\zeta_{i}\right)$ over $Q\left(\zeta_{j}\right)$. Then $G_{j}=\bigcup_{\lambda=1}^{p-1} G_{j / 1} \bullet \sigma_{\lambda}$ and $\nu^{\prime}+\nu \mu \equiv \nu^{\prime}+\nu \lambda(\bmod p)$ for any element $\sigma_{\mu}$ of $G_{j / 1} \cdot \sigma_{\lambda}$. This shows that

$$
\left[K_{j, \nu}\right] \mathrm{a}_{i, \nu^{\prime}}^{*}=\sum_{\lambda=1}^{p-1}\left(\prod_{\sigma_{\mu} \in G_{J} / \cdot \sigma \lambda} \mathfrak{a}^{\sigma} \nu_{\mu}\right)_{i, \nu^{\prime}+\nu \lambda}^{*} .
$$

The case $i=j$. For each $\mu$ such that $\mu \neq-1(\bmod p)$, let $x_{\mu}$ be an integer such that $(1+\mu) x_{\mu} \equiv 1\left(\bmod p^{i}\right)$. Then Theorem 3.2 and Proposition 3.3 imply 
that

$$
\begin{aligned}
{\left[K_{i, \nu}\right] a_{i, \nu^{\prime}}^{*} } & =\sum_{\substack{\left.\rho \mu \in G_{i} \\
\mu \neq-1 \bmod \nu^{\prime}\right)}}\left(a^{s} x_{\mu} ; p^{i-1}\left(\nu^{\prime}+\nu \mu\right) x_{i \mu}, 1\right)^{*} \\
& +\sum_{\substack{\alpha \mu \in G_{i} \\
\mu \equiv=-1(\bmod p)}}\left(\mathfrak{a} ; p^{i-1}\left(\nu^{\prime}+\nu / \mu\right), 1+\mu\right)^{*} .
\end{aligned}
$$

In the first term of the right hand side, $\sigma_{x_{\mu}}$ ranges over $\bigcup_{\lambda=2}^{\nu-1} G_{i / 1} \cdot \sigma_{\lambda}$ and $(\nu+\nu \mu) x_{\mu} \equiv\left(\nu^{\prime}-\nu\right) \lambda+\nu(\bmod p)$ for any $\sigma_{x_{\mu}}$ of $G_{i / 1} \cdot \sigma_{\lambda}$. Then the first term of (4.1) is equal to

$$
\sum_{\lambda=2}^{p-1}\left(\prod_{\sigma \in G_{i} / \cdot \sigma \lambda} \mathfrak{a}^{\sigma}\right)_{i,\left(\nu^{\prime}-\nu\right) \lambda+\nu}^{*}=\sum_{\lambda=2}^{p-1}\left(N_{i / 1}(a)^{\Omega \lambda}\right)_{i,\left(\nu^{\prime}-\nu\right) \lambda+\nu}^{*}
$$

In particular, if $\nu^{\prime}=\nu$, this is equal to $-\left(N_{i / 1}(\mathfrak{a})\right)_{i, \nu}^{*}$. In the second term of (4.1), let $p^{h}$ be the highest power of $p$ which divides $1+\mu$ and set $1+\mu=\mu_{h} \cdot p^{h}$. Then (3.1) implies that

$$
\left(a ; p^{i-1}\left(\nu^{\prime}+\nu \mu\right), 1+\mu\right)^{*}=\left(N_{i / i-h}(a) ; p^{i-h-1}\left(\nu^{\prime}-\nu\right), \mu_{h}\right)^{*}
$$

since the ideal class of $\mathfrak{a}$ as a $Z\left[\zeta_{i-h}\right]$-module is the norm $N_{i / i-h}(\mathfrak{a})$ of a from $Z\left[\zeta_{i}\right]$ to $Z\left[\zeta_{i-h}\right]$ ([1]). When $\sigma_{\mu}$ ranges over elements of $G_{i}$ such that $1+\mu \equiv 0$ $\left(\bmod p^{k_{1}}\right)$ and $1+\mu \neq \equiv 0\left(\bmod p^{n+1}\right), \sigma_{\mu_{h}}^{-1}$ obviously ranges over the elements of $G_{i-h}=\bigcup_{\lambda=1}^{p-1} G_{i-h / 1} \cdot \sigma_{\lambda}$ and $\left(\nu^{\prime}-\nu\right) \gamma \equiv\left(\nu^{\prime}-\nu\right) \lambda(\bmod p)$ for any or of $G_{i-h / 1} \cdot \sigma_{\lambda}$. Hence the second term of (4.1) is equal to

$$
\begin{aligned}
& \sum_{h=1}^{i-1} \sum_{\lambda=1}^{p-1}\left(\prod_{\sigma \in G_{i}-h / 1 \cdot \sigma \lambda} N_{i / i-h}(a)^{\sigma}\right)_{i-h,\left(\nu^{\prime}-\nu\right) \lambda}^{*}+\left(N_{i / 1}(a) ; \nu^{\prime}-\nu, 0\right)^{*} \\
= & \sum_{n=1}^{i-1} \sum_{\lambda=1}^{p-1}\left(N_{i / 1}(a)^{\pi \lambda}\right)_{i-h,\left(\nu^{\prime}-\nu\right) \lambda}^{*}+\left(N_{i / 1}(a) ; \nu^{\prime}-\nu, 0\right)^{*},
\end{aligned}
$$

where if $\nu^{\prime} \neq \nu,\left(N_{i / 1}(\mathfrak{a}) ; \nu^{\prime}-\nu, 0\right)^{*}=\left(N_{i / 1}(\mathfrak{a})^{\sigma_{\nu^{\prime}-\nu}^{-1}}\right)_{0}^{*}$ and if $\nu^{\prime}=\nu,\left(N_{i / 1}(\mathfrak{a}) ; \nu^{\prime}-\right.$ $\nu, 0)^{*}=0$ and $\sum_{\lambda=1}^{p-1}\left(N_{i / 1}(\mathfrak{a})^{\alpha \lambda}\right)_{i-h,\left(\nu^{\prime}-\nu\right) \lambda}^{*}=\left(N_{i / 0}(a)\right)_{i-h, 0}^{*}=0$ since $N_{i / 0}(a)$ is a principal ideal. The case $i<j$. From Theorem 3.2 we have

$$
\left[K_{j, \nu}\right] a_{i, \nu^{\prime}}^{*}=\sum_{\sigma \mu \in G_{i}}\left(\tilde{a} ; p^{j-1}\left(\nu^{\prime}+\nu \mu\right), p^{j-i}+\mu\right)^{*} .
$$

Let $x_{\mu}$ be an integer such that $\left(p^{j-i}+\mu\right) x_{\mu} \equiv 1\left(\bmod p^{j}\right)$. Then $\left(\tilde{a} ; p^{j-1}\left(\nu^{\prime}+\right.\right.$ $\left.\nu \mu), p^{j-i}+\mu\right)^{*}=\left(\tilde{a}^{3} x_{\mu}\right)_{j, \nu}^{*} x_{\mu+\nu}$ by Proposition 3.3, $\sigma_{x_{\mu}}$ ranges over the elements of $G_{i}$, and $\nu^{\prime} x_{\Lambda}+\nu \equiv \nu^{\prime} \lambda+\nu(\bmod p)$ for any $\sigma_{x_{\mu}}$ of $G_{i / 1_{1}} \cdot \sigma_{\lambda}$. This shows that

$$
\left[K_{j, \nu}\right] \mathrm{a}_{i, \nu^{\prime}}^{*}=\sum_{\lambda=1}^{p-1}\left(\prod_{\sigma \in Q_{i / 1}, \Omega_{\lambda}} \tilde{a}^{\Im}\right)_{j, \nu^{\prime} \lambda+\nu}^{*}=\sum_{\lambda=1}^{\nu-1}\left(\widetilde{N_{i / 1}}(\mathfrak{a})^{\wedge \lambda}\right)_{j, \nu \lambda+\nu}
$$


Summalizing, we have

Proposition 4.1. Let $\left(B\right.$ be an abelian group of type $\left(p, p^{e}\right)$. Then $G(Q \otimes)$ acts on $\phi\left(C_{0}(\mathrm{D})\right)$ as follows.

1. [Q] acts trivially.

2. $\left[K_{0}\right] \mathfrak{a}_{0}^{*}=-\mathfrak{a}_{0}^{*}$.

3. $\left[K_{0}\right] a_{i, \nu}^{*}=\sum_{\mu=0, \neq \nu}^{p-1} a_{i, \mu}^{*}$.

4. $\left[K_{i, \nu}\right] \mathfrak{a}_{0}^{*}=\sum_{\lambda=1}^{p-1}\left(\tilde{\mathfrak{a}}^{\gamma \lambda}\right)_{i, \nu+\lambda}^{*}$.

5. $\left[K_{j, \nu}\right] a_{i, \nu}^{*}$

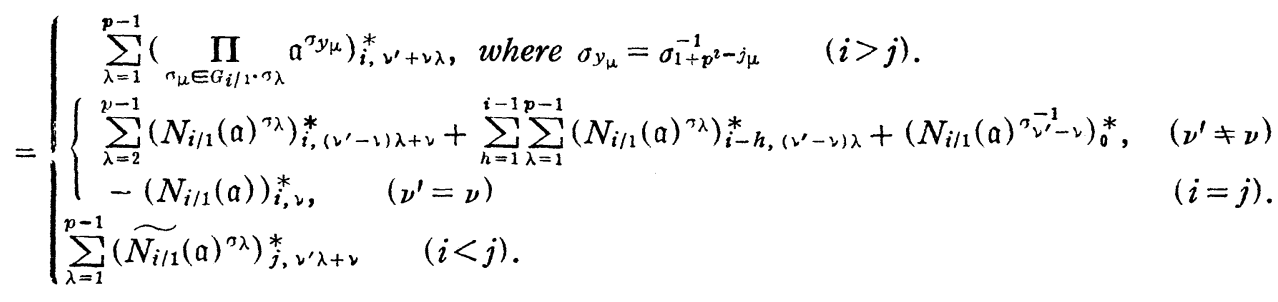

\section{BIBLIOGRAPHY}

[1] E. Artin: Questions de base minimale dans la théorie de nombres algébriques, Colloq. Int. Cent. Nat. Rech. Sci. 24 (1950), 19-20.

[2] H. Cartan and S. Eilenberg: Homological algebra, Princeton University Press, 1956.

[3] C. W. Curtis and I. Reiner: Representation theory of finite groups and associative algebras, Int. Sci. Press, 1962.

[4] D. S. Rim: On projective class groups, Trans. Amer. Math. Soc. 98 (1961), 459-467.

[5] R. G. Swan: Induced representations and projective modules, Ann. Math. 71 (1960), 552-578.

[6] R. G. Swan: The Grothendieck ring of a finite group, Topology, vol. 2 (1963), 85-110.

Tokyo University of Education 